\title{
Advanced Hepatocellular Carcinoma in A Scottish Population - Current Experience and the Future
}

\author{
Baxter MA ${ }^{1,2 *}$, Peters AL $^{1}$, Graham J ${ }^{1,3}$ \\ ${ }^{1}$ Beatson West of Scotland Cancer Centre, Glasgow, UK \\ ${ }^{2}$ University of Dundee, UK \\ ${ }^{3}$ University of Glasgow, UK
}

Received: 阱 August 07, 2018; Published: 制 August 10, 2018

*Corresponding author: Dr Mark A Baxter, Department of Medical Oncology, Beatson West of Scotland Cancer Centre, 1053 Great Western Road, Glasgow, UK

\begin{abstract}
Sorafenib is the current first line standard of care in patients with advanced hepatocellular carcinoma (HCC). Several other drugs are emerging as efficacious agents in this setting. This article will outline our current real-world experience with sorafenib in a Scottish population as well as discuss the evidence behind new potential therapies.
\end{abstract}

Keywords: Sorafenib; Hepatocellular carcinoma; HCC

Abbreviations: HCC: Hepatocellular Carcinoma; PS: Performance Status; OS: Overall Survival; TACE: Transarterial Chemoembolization; VEGF: Vascular Endothelial Growth Factor; PDGFR: Platelet Derived Growth Factor Receptor; FGFR: Fibroblast Growth Factor Receptor; WoS: West of Scotland

\section{Introduction}

Hepatocellular carcinoma (HCC) is the fifth most common cancer affecting patients worldwide and accounts for $2 \%$ of cancer cases in the United Kingdom [1,2]. Incidence is rising and it is associated with several known risk factors including heavy alcohol consumption, non-alcoholic steatohepatitis and chronic hepatitis B and $\mathrm{C}$ infection $[3,4]$. If detected at an early stage, HCC is potentially curable. Unfortunately, many patients are diagnosed at an advanced stage (according to the Barcelona Clinic Liver Cancer criteria) and treatment is therefore palliative focusing on prolonging survival and improving symptom burden. In the advanced setting, sorafenib is recommended as first line option in patients who cannot benefit from resection, transplantation, ablation or transarterial chemoembolization (TACE), and who still have preserved liver function [5]. Sorafenib is the only drug that has shown a significant survival benefit in the last decade. It is a multi-kinase inhibitor with particular action against VEGFR2, PDGFR, FGFR1, Raf-1, B-Raf and c-Kit receptors [6].

In 2008 and 2009, the SHARP and Asia-Pacific trials demonstrated a significant survival benefit for sorafenib versus placebo of 2.8 months and 2.3 months $[7,8]$. The toxicity profile was acceptable and on the basis of these studies, sorafenib was licenced for use in advanced HCC in many countries including Scotland. Since then, several agents have failed to show superiority or noninferiority when compared to Sorafenib in the first-line setting [9], with only a handful having an impact on survival. Sorafenib therefore remains the current mainstay of treatment worldwide and at our institution. As trial populations are recognised to not always be representative of a real-world population [10], we sought to determine whether the experience at our institution correlated with the results reported by the SHARP and Asia-Pacific studies.

\section{Results and Discussion}

We retrospectively identified 55 patients at our institution who received sorafenib between November 2016 and October 2017. Baseline demographic data were recorded, and electronic patient records were reviewed for duration of treatment, treatment tolerance and survival data. In our study population, 55 patients received sorafenib during the time period. The demographics compared to the SHARP study are shown in Table 1. 46 (84\%) were male and $9(16 \%)$ female. The median age was 68 (Range 37-84). All patients were Childs-Pugh A. On starting treatment, performance status (PS) was 0 in 49\%, 1 in $47 \%$ and 2 in $4 \%$. At the time of analysis, 22 (40\%) patients were still alive and 7 (13\%) 
patients were still on treatment. The median number of completed treatments was 3 with a median duration of treatment of $5.8 \mathrm{~m}(95 \%$ CI: $2.0 \mathrm{~m}-9.6 \mathrm{~m})$. Sorafenib was stopped due to toxicity in 11 (20\%) patients, compared to $38 \%$ reported in SHARP. The median overall survival was $10.4 \mathrm{~m}$ ( $95 \% \mathrm{CI}$ : 5.6m-15.2m), with no significant different between PS 0 and PS $1(11.1 \mathrm{~m} v 9.8 \mathrm{~m}, \mathrm{p}=0.8)$. There was no association between baseline alpha-fetoprotein and survival. Overall, the patients treated with sorafenib at our institution appear to have similar duration of treatment and overall survival compared to that reported in SHARP (Tables $1 \& 2$ ). The obvious limitations in this study are the small numbers and the retrospective nature. Nevertheless, the two populations appear to have broadly similar baseline characteristics and the comparable duration of treatment and overall survival is encouraging, supporting the use of sorafenib in our West of Scotland population.

Table 1: Demographic data in our West of Scotland population (WoS) compared to the SHARP study population.

\begin{tabular}{|c|c|c|}
\hline & WoS (n=55) & SHARP (n=299) \\
\hline Sex & & \\
\hline Male & $84 \%$ & $87 \%$ \\
\hline Female & $16 \%$ & $13 \%$ \\
\hline Age & 68 (range 37-84) & 64.9 \\
\hline PS & $49 \%$ & $54 \%$ \\
\hline 0 & $47 \%$ & $38 \%$ \\
\hline 1 & $4 \%$ & $8 \%$ \\
\hline 2 & 5 (range 0- 56) & 5 \\
\hline $\begin{array}{c}\text { Median cycles of sorafenib } \\
\text { completed }\end{array}$ & & \\
\hline
\end{tabular}

Table 2: Median duration of treatment (DoT) and median overall survival (OS) in our population compared to the SHARP population. $(\mathrm{m})=$ months.

\begin{tabular}{|c|c|c|}
\hline & WoS $(\mathbf{n}=\mathbf{5 5})$ & SHARP $(\mathbf{n}=\mathbf{2 9 9})$ \\
\hline Median DoT & $5.8 \mathrm{~m}(2.0-9.6)$ & $5.3 \mathrm{~m}(\mathrm{NR})$ \\
\hline Median OS & $10.4 \mathrm{~m}(5.6-15.2)$ & $10.7 \mathrm{~m}(9.4-13.3)$ \\
\hline
\end{tabular}

As mentioned in the introduction, little progress has been made in the treatment of advanced HCC since the SHARP trial. Multiple agents targeting various pathways have been tested unsuccessfully in the first line including multikinase inibitors (brivanib, sunitinib and linifanib [11-13], anti-EGFR agents (Erlotinib) [14] and cytotoxics such as doxorubicin, 5-flurouracil and oxaliplatin $[15,16]$. There is therefore an obvious need for patients who are unable to tolerate, or progress on sorafenib therapy to have treatment options. This need has prompted investigation of several agents as first- or second-line treatment options for advanced HCC, with varying degrees of success in recent years. The multi-kinase inhibitors lenvatinib, regorafenib and carbozantinib have all shown significant promise. Of note, they all have similar modes of action to sorafenib. In the REFLECT study, lenvatinib was the first drug to show non-inferiority to sorafenib in the first line setting with a mOS of 13.6 months (95\% CI $12 \cdot 1-14 \cdot 9)$ versus (12.3 months,
10.4-13.9; hazard ratio $0 \cdot 92,95 \%$ CI $0 \cdot 79-1 \cdot 06$ ) [17], while in the RESOURCE study regorafenib showed a survival advantage over placebo following progression on sorafenib. Median survival was $10 \cdot 6$ months (95\% CI 9.1-12·1) for regorafenib versus $7 \cdot 8$ months (95\% CI 6.3-8.8) for placebo [18]. A further multi-kinase inhibitor, carbozantinib is currently being tested in the Phase III CELESTIAL trial following promising Phase II results [19].

Unfortunately, other novel agents have been less successful including the mTOR inhibitor everolimus, the anti-metabolite ADI-PEG20 and the anti-angiogenic Cediranib (AZD2171) [2022]. With multi-kinase inhibitors being the main success stories in the treatment of HCC, the emergence of immune checkpoint inhibitors has sparked interest in a new mechanism to target HCC. This is supported by preliminary results from a Phase I/II study of nivolumab, reporting a partial response rate of $19 \%$ and 1-year OS of $62 \%$ [23]. The study is ongoing, and the results are eagerly anticipated. If the results from other tumour sites are replicated in advanced HCC, it could herald a new and exciting treatment landscape.

\section{Conclusion}

Sorafenib continues to be the standard of care in advanced HCC. Our real-world data support the findings of the SHARP study and the use of sorafenib in this population. The landscape of HCC treatments may change in the near future with the emergence of immune checkpoint inhibitors.

\section{References}

1. Ferlay J, Soerjomataram I, Dikshit R (2015) Cancer incidence and mortality worldwide: sources, methods and major patterns in GLOBOCAN 2012. International journal of cancer 136(5): E359-386.

2. Cruk (2018) Liver Cancer Incidence Statistics.

3. Forner A, Llovet JM, Bruix J (2012) Hepatocellular carcinoma. Lancet 379(9822): 1245-1255.

4. Piscaglia F, Svegliati-Baroni G, Barchetti A (2016) Clinical patterns of hepatocellular carcinoma in nonalcoholic fatty liver disease: A multicenter prospective study. Hepatology 63(3): 827-838.

5. (2012) EASL-EORTC Clinical Practice Guidelines: Management of hepatocellular carcinoma. Journal of Hepatology 56(4): 908-943.

6. Shin JW, Chung YH (2013) Molecular targeted therapy for hepatocellular carcinoma: current and future. World journal of gastroenterology 19(37): 6144-6155.

7. Llovet JM, Ricci S, Mazzaferro V (2008) Sorafenib in advanced hepatocellular carcinoma. The New England journal of medicine 359(4): 378-390.

8. Cheng AL, Kang YK, Chen Z (2009) Efficacy and safety of sorafenib in patients in the Asia-Pacific region with advanced hepatocellular carcinoma: a phase III randomised, double-blind, placebo-controlled trial. The Lancet. Oncology 10(1): 25-34.

9. Desai JR, Ochoa S, Prins PA, He AR (2017) Systemic therapy for advanced hepatocellular carcinoma: an update. Journal of Gastrointestinal Oncology 8(2): 243-255.

10. Kennedy Martin T, Curtis S, Faries D, Robinson S, Johnston J (2015) A literature review on the representativeness of randomized controlled trial samples and implications for the external validity of trial results. Trials 16: 495. 
11. Johnson PJ, Qin S, Park JW et al (2013) Brivanib versus sorafenib as first-line therapy in patients with unresectable, advanced hepatocellular carcinoma: results from the randomized phase III BRISK-FL study. Journal of clinical oncology 31(28): 3517-3524.

12. Cheng AL, Kang YK, Lin DY (2013) Sunitinib versus sorafenib in advanced hepatocellular cancer: results of a randomized phase III trial. Journal of clinical oncology 31(32): 4067-4075.

13. Cainap C, Qin S, Huang WT (2015) Linifanib versus Sorafenib in patients with advanced hepatocellular carcinoma: results of a randomized phase III trial. Journal of clinical oncology: 33(2): 172-179.

14. Zhu AX, Rosmorduc O, Evans TR (2015) SEARCH: a phase III, randomized, double-blind, placebo-controlled trial of sorafenib plus erlotinib in patients with advanced hepatocellular carcinoma. Journal of clinical oncology 33(6): 559-566.

15. Abou Alfa Gk ND, Knox Jj (2016) Phase III randomized study of sorafenib plus doxorubicin versus sorafenib in patients with advanced hepatocellular carcinoma (HCC): CALGB 80802 (Alliance). Journal of clinical oncology 34 Abstract 192.

16. Qin S, Bai Y, Lim HY (2013) Randomized, multicenter, open-label study of oxaliplatin plus fluorouracil/leucovorin versus doxorubicin as palliative chemotherapy in patients with advanced hepatocellular carcinoma from Asia. Journal of clinical oncology: official journal of the American Society of Clinical Oncology 31(28): 3501-3508.

17. Kudo M, Finn RS, Qin S et al (2018) Lenvatinib versus sorafenib in firstline treatment of patients with unresectable hepatocellular carcinoma: a randomised phase 3 non-inferiority trial. Lancet 391(10126): 11631173.

18. Bruix J, Qin S, Merle P (2017) Regorafenib for patients with hepatocellular carcinoma who progressed on sorafenib treatment (RESORCE): a randomised, double-blind, placebo-controlled, phase 3 trial. Lancet 389(10064): 56-66.

19. Verslype C CA, Kelley Rk (2012) Activity of cabozantinib (XL184) in hepatocellular carcinoma: Results from a phase II randomized discontinuation trial (RDT). Journal of clinical oncology $30 \mathrm{Abstr} 4007$.

20. Alberts SR, Fitch TR, Kim GP (2012) Cediranib (AZD2171) in patients with advanced hepatocellular carcinoma: a phase II North Central Cancer Treatment Group Clinical Trial. American journal of clinical oncology 35(4): 329-333.

21. Yang TS, Lu SN, Chao Y (2010) A randomised phase II study of pegylated arginine deiminase (ADI-PEG 20) in Asian advanced hepatocellular carcinoma patients. British journal of cancer 103(7): 954-960.

22.Zhu AX, Kudo M, Assenat E (2014) Effect of everolimus on survival in advanced hepatocellular carcinoma after failure of sorafenib: the EVOLVE-1 randomized clinical trial. Jama 312(1): 57-67.

23. Squibb BM (2016) A study to evaluate the effectiveness, safety and tolerability of nivolumab and the combination nivolumab plus ipilimumab in patients with advanced liver cancer (CheckMate040).

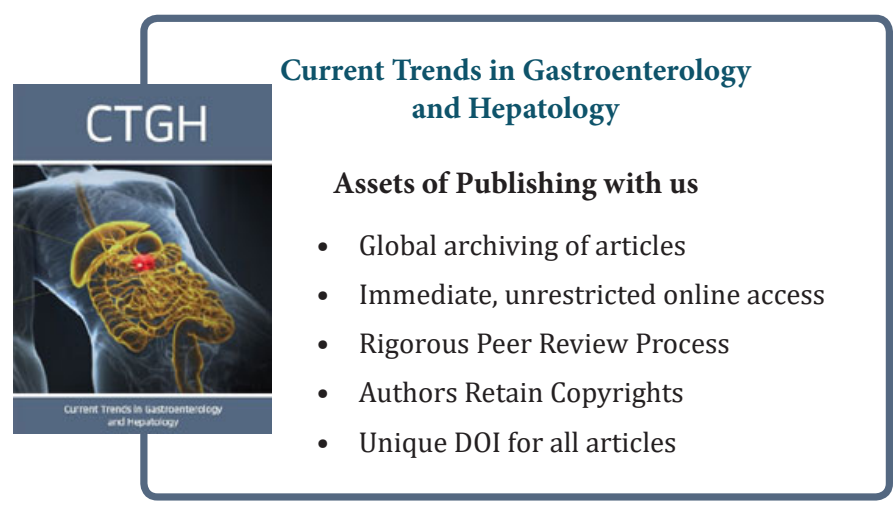

